\title{
Expression of mTOR Signaling Pathway Molecules in Triple-Negative Breast Cancer
}

\author{
Kei Ito ${ }^{a, b}$ Hideaki Ogatac Naoko Honma ${ }^{a}$ Kazutoshi Shibuyad \\ Tetuo Mikami ${ }^{a}$ \\ ${ }^{a}$ Department of Pathology, Toho University Graduate School of Medicine, Tokyo, Japan; ${ }^{b}$ Department of Medical \\ Technology, Faculty of Health Sciences, Tukuba International University, Ibaraki, Japan; ' Department of Surgery, \\ Toho University Omori Medical Center, Tokyo, Japan; ${ }^{\mathrm{d}}$ Department of Surgical Pathology, Toho University Omori \\ Medical Center, Tokyo, Japan
}

\section{Keywords}

Triple-negative breast cancer · mTOR · Glucose transporter

\begin{abstract}
Introduction: Triple-negative breast cancer (TNBC), which lacks expression of estrogen receptor (ER), progesterone receptor $(\mathrm{PgR})$, and epidermal growth factor receptor 2 (HER2), currently has no effective hormonal or molecular target therapy. Objective and Methods: To elucidate the role of the mammalian target of rapamycin (mTOR) signaling pathway in TNBC, the expression of molecules involved in mTOR signaling including mTOR, phosphorylated (p)mTOR, p-4EBP1, GLUT1, GLUT3, HIF-1a, and Ki67 was investigated by immunohistochemistry in 35 TNBC and 81 nonTNBC cases. Results: Expression of p-mTOR, the activated form of mTOR, but not unphosphorylated MTOR, was significantly higher in non-TNBC cases than in TNBC cases. Expression of $\mathrm{p}-4 \mathrm{EBP} 1, \mathrm{GLUT1}$, and GLUT3 was higher in TNBC cases than in non-TNBC cases. When the localization of $p$ mTOR was classified as nuclear, perinuclear, or cytoplasmic, nuclear localization of p-mTOR was observed more frequently in TNBC than in non-TNBC cases and was correlated with the expression of GLUT1 and GLUT3, which was related
\end{abstract}

to proliferation activity examined with Ki67. Conclusions: mTOR signaling regulates cell proliferation in some cases of TNBC and may be a potential target of molecular therapy for TNBC.

(c) 2019 S. Karger AG, Basel

\section{Introduction}

Triple-negative breast cancer (TNBC) is defined as a tumor without expression of estrogen receptor (ER), progesterone receptor (PgR), or epidermal growth factor receptor 2 (HER2). Because the differentiation and proliferation of TNBC cancer cells are independent of hormonal regulation and the HER2 signaling pathway, no effective therapy has been established to treat this subgroup of breast cancer patients [1]. Alternative signaling pathways governing tumor cell biology will have to be elucidated to identify new therapeutic targets and biomarkers for the early detection of TNBC tumors. Here, we investigated the expression of mammalian target of rapamycin (mTOR) signaling pathway (one of the cell growth pathways involved in the uptake of sugar and amino acids) molecules in TNBC.

\section{KARGER}

(c) 2019 S. Karger AG, Basel

E-Mail karger@karger.com

www.karger.com/pat 
mTOR is a serine/threonine kinase that controls transcription, translation, and autophagy to positively regulate growth factors, metabolism, and survival [2]. Although several drugs that inhibit mTOR have undergone clinical trials for the treatment of breast cancer [3], including TNBC [4], targeted therapies for TNBC remain unavailable [5]. mTOR is a component of the complex known as mTORC1, which also includes mLST8 (GßL) and Raptor (regulatory-associated-protein of mTOR) and promotes protein translation through a variety of interactions. One such mechanism is phosphorylation of the key translational regulators p70 ribosomal S6 kinase (S6K) and eukaryotic initiation factor 4E-binding protein 1 (4EBP1) by phosphorylated (p)-mTOR, the activated form of mTOR. Under basal conditions, $\mathrm{S} 6 \mathrm{~K}$ is bound to the eukaryotic initiation factor 3 (eIF3) translation initiation complex. mTORC1 activity promotes the phosphoactivation of $\mathrm{S} 6 \mathrm{~K}$, which results in its dissociation from eIF3 [6] and enhances the translation of mRNAs bearing $5^{\prime}$-terminal oligopyrimidine tracts, such as those encoding ribosomal proteins [7, 8]. Phosphorylation of 4EBP1(p-4EBP1) increases mRNA translation by inhibiting the interaction between 4EBP1 and eukaryotic initiation factor 4 (eIF4), thereby releasing a block to the formation of the translation initiation complex [9]. mTOR also acts as a positive regulator of hypoxia inducible factor (HIF-1), a transcription factor that regulates many genes involved in tumor-induced angiogenesis [10]. HIF-1 enhances glycolytic metabolism by promoting the expression of glucose transporters [11]. It has been reported that HIF-1 and glucose transporter 1 (GLUT1) protein expression levels are diminished with rapamycin treatment [12]. The expression of GLUT3 has also been shown to depend on mTOR activity, and to require the transcription factor HIF-1 $\alpha$ [13].

In this study, we have compared the expression of mTOR signaling pathway molecules in TNBC and nonTNBC, with the aim of identifying potential therapeutic and diagnostic markers targeted specifically to TNBC.

\section{Materials and Methods}

\section{Patient Selection}

One hundred and sixteen invasive ductal carcinomas surgically resected at Toho University Medical Center Omori Hospital from 2008 to 2010 were collected. Cases of lobular carcinoma and other special histological types were omitted. Cases receiving preoperative chemotherapy and radiation therapy were also omitted. The cases were routinely examined for the expression of ER, PgR, and HER2, using the following primary antibodies and staining kit: anti-ER (Nichirei, Tokyo, Japan), anti-PgR (Thermo Scientific,
Table 1. Clinicopathological features of breast cancer in this study

\begin{tabular}{lcll}
\hline & $\begin{array}{l}\text { TNBC } \\
(n=35)\end{array}$ & $\begin{array}{l}\text { Non-TNBC } \\
(n=81)\end{array}$ & $p$ \\
\hline Age, years & $62 \pm 14$ & $61 \pm 14$ & $\mathrm{~ns}$ \\
Males & 0 & 0 & $\mathrm{~ns}$ \\
Females & 35 & 81 & $\mathrm{~ns}$ \\
Tumor size, mm & $32 \pm 20$ & $20 \pm 12$ & $<0.01^{\mathrm{b}}$ \\
Lymph node status & & & $\mathrm{ns}$ \\
$\quad$ Positive for metastasis & 14 & 15 & \\
$\quad$ Negative for metastasis & 19 & 57 & \\
Hormone receptor-positive & $0(0 \%)$ & $72(89 \%)$ & \\
(ER or PgR) & & & \\
HER2-positive & $0(0 \%)$ & $14(17 \%)$ & \\
Nuclear grade [21] & & & \\
$\quad 1$ & 1 & 36 & $<0.01^{\mathrm{a}}$ \\
$\quad 2$ & 10 & 24 & \\
3 & 24 & 21 & \\
Histological grade & & & \\
$\quad 1$ & 1 & 22 & \\
2 & 13 & 44 & \\
3 & 21 & 15 & \\
\hline
\end{tabular}

Values express $n$ or mean \pm SD. ns, not significant; TNBC, triple-negative breast cancer; ER, estrogen receptor; PgR, progesterone receptor.

a Statistically significant, $\chi^{2}$ test; ${ }^{b}$ statistically significant, MannWhitney U test.

Waltham, MA, USA), and HercepTest (Dako, Carpinteria, CA, USA). For ER and PgR, cases were considered positive when $>1 \%$ of cancer cells showed positive immunoreactivity. For HercepTest, cases were considered negative if they scored 0 or +1 , and positive if they scored +3 . Weakly positive cases $(2+)$ were considered equivocal and were further evaluated by HER2 gene amplification using the FISH method. In this study, FISH was performed for 2 cases, neither of which showed HER2 gene amplification. When all 3 markers were negative, the cases were considered to be TNBC. By these criteria, a total of 35 cases were classified as TNBC and 81 as non-TNBC. The clinicopathological characteristics of the TNBC and non-TNBC tumors are summarized in Table 1. Prognostic information was collected from medical charts. The mean follow-up time was 71 (range 1-123) months.

\section{Immunohistochemistry}

We examined formalin-fixed and paraffin-embedded $4-\mu \mathrm{m}$ sections from 1 representative paraffin block per case for mTOR, p-mTOR, p-4EBP1, GLUT1, GLUT3, HIF-1 $\alpha$, and Ki67. The sources of the antibodies for these antigens are summarized in Table 2. Endogenous peroxidase was blocked with $0.5 \%$ hydrogen peroxide in methanol for $30 \mathrm{~min}$. Before the primary antibody was applied, slides were subjected to antigen retrieval (Table 2). After application of the primary antibodies, the slides were washed with PBS and then incubated with secondary EnVision+System-HRPlabeled polymer (Dako). Finally, the tissue sections were visualized with Stable DAB (Falma, Tokyo, Japan) and counterstained with Mayer's hematoxylin. 
Table 2. Antibodies used and their conditions of immunostaining

\begin{tabular}{|c|c|c|c|c|}
\hline Antigen & Antibody & Dilution & Antigen retrieval (buffer) & Supplier \\
\hline $\begin{array}{l}\text { mTOR } \\
\text { phospho-mTOR }\end{array}$ & rabbit, monoclonal & $\times 50$ & microwave for $20 \min (\mathrm{pH} 6)^{\mathrm{a}}$ & Cell Signaling Technology, Danvers, MA, USA \\
\hline $\begin{array}{l}\text { (Ser2448) } \\
\text { phospho-4E-BP1 }\end{array}$ & rabbit, monoclonal & $\times 100$ & microwave for $20 \min (\mathrm{pH} 6)^{\mathrm{a}}$ & Cell Signaling Technology \\
\hline (Thr37/46) & rabbit, monoclonal & $\times 1,000$ & autoclave for $10 \mathrm{~min}(\mathrm{pH} 6)^{\mathrm{b}}$ & Cell Signaling Technology \\
\hline GLUT1 & rabbit, polyclonal & $\times 200$ & microwave for $10 \min (\mathrm{pH})^{\mathrm{a}}$ & Abcam, Cambridge, UK \\
\hline GLUT3 & rabbit, polyclonal & $\times 200$ & microwave for $10 \min (\mathrm{pH} 6)^{\mathrm{a}}$ & Abcam \\
\hline HIF-1a & mouse, polyclonal & $\times 100$ & autoclave for $10 \min (\mathrm{pH} 9)^{\mathrm{b}}$ & BD Transduction Laboratories, San José, CA, USA \\
\hline Ki67 & rabbit, polyclonal & $\times 100$ & autoclave for $10 \min (\mathrm{pH} 6)^{\mathrm{b}}$ & Thermo Scientific, Waltham, MA, USA \\
\hline
\end{tabular}

${ }^{\mathrm{a}}$ Microwave of $500 \mathrm{~W}$ was used for antigen retrieval; ${ }^{\mathrm{b}}$ autoclave for antigen retrieval was performed at $120^{\circ} \mathrm{C}$.

Both the intensity and the area of immunohistochemical staining in the cancer tissue sections were evaluated, and the immunoreactivity score was calculated. The proportion of areas exhibiting positive staining was scored as follows: 1 (focal), $1-10 \% ; 2$ (partial), 11-30\%; and 3 (diffuse), $>30 \%$. The staining intensity score, ranging from 0 to 3 , was evaluated as follows: 0 , no staining; 1 , weak; 2 , moderate; and 3, strong. The multiplication of the proportion score and the intensity score was taken as the immunoreactivity score.

Subsequently, the cellular localization of p-mTOR was examined, and the cases were classified into 3 categories: nuclear, perinuclear, and cytoplasmic expression. Ki67 expression was also evaluated in $>500$ cancer cells, and the proportion of Ki67-positive cells (the number of Ki67 positive cells/number of total cancer cells) was calculated as a percentage.

\section{Statistical Analysis}

Spearman's rank correlation test, the Mann-Whitney U test, and the $\chi^{2}$ test with $R$ software v3.2.2 were used to assess statistical significance. Prognosis was analyzed by the Kaplan-Meier method along with the log rank test. $p<0.05$ was considered statistically significant.

\section{Results}

\section{Expression of Each Molecule}

In normal mammary glands, mTOR showed weak cytoplasmic expression in epithelial cells in mammary ducts and lobules. On the other hand, the p-mTOR level varied; it was detected in the cytoplasm of epithelial cells in some cases but not others. The expression of Nuclear p-4EBP1 and membranous GLUT3 was low or negative. There was no GLUT1 expression in the epithelial cells of normal mammary glands, but high expression in myoepithelial cells was observed in a few cases.

In both the TNBC and non-TNBC cases, expression of mTOR was observed in the cytoplasm, GLUT1 and
GLUT3 at the cell membrane, and p-4EBP1 and HIF-1a in the nucleus, and p-mTOR in both the cytoplasm and nucleus (Fig. 1). p-mTOR expression was first evaluated in the whole cell without considering subcellular localization. Expression of p-mTOR was significantly lower in TNBC than in non-TNBC, but mTOR expression was not significantly different between the tumor types. In contrast to p-mTOR, the expression of p-4EBP1, GLUT1, and GLUT3 was significantly higher in TNBC than in non-TNBC cases (Fig. 2). A positive weak correlation of expression was observed between GLUT1 and p-4EBP1 $(\rho=0.246, p=0.0079)$, and between GLUT3 and $\mathrm{p}-4 \mathrm{EBP} 1$ $(\rho=0.267, p=0.0037)$. In addition, the expression of GLUT1 was positively correlated with that of HIF-1 $\alpha$ $(\rho=0.336, p=0.0002)$ and GLUT3 $(\rho=0.461, p<0.0001)$ (Table 3).

To identify whether the expression of mTOR, pmTOR, p-4EBP1, GLUT1, GLUT3, and HIF-1 $\alpha$ was related to prognostic factors, the correlation with clinicopathological factors such as the age of the patient, tumor size, nuclear grade, histological grade, lymph node status, hormone receptor status, and HER2 overexpression was tested. However, no significant relationship between the expression of these proteins and the clinicopathological factors was observed. There was also no significant correlation with patient prognosis (overall survival). Specifically, overall survival was compared in patients with high and low expression of p-mTOR among 116 cases, but no significant difference was found (online suppl. Fig. 1; for all online suppl. material, see www.karger.com/doi/10.1159/000503311). Among the TNBC cases, no significant difference in prognosis was observed between the cases with nuclear p-mTOR expression and those with perinuclear or cytoplasmic expression (data not shown). 

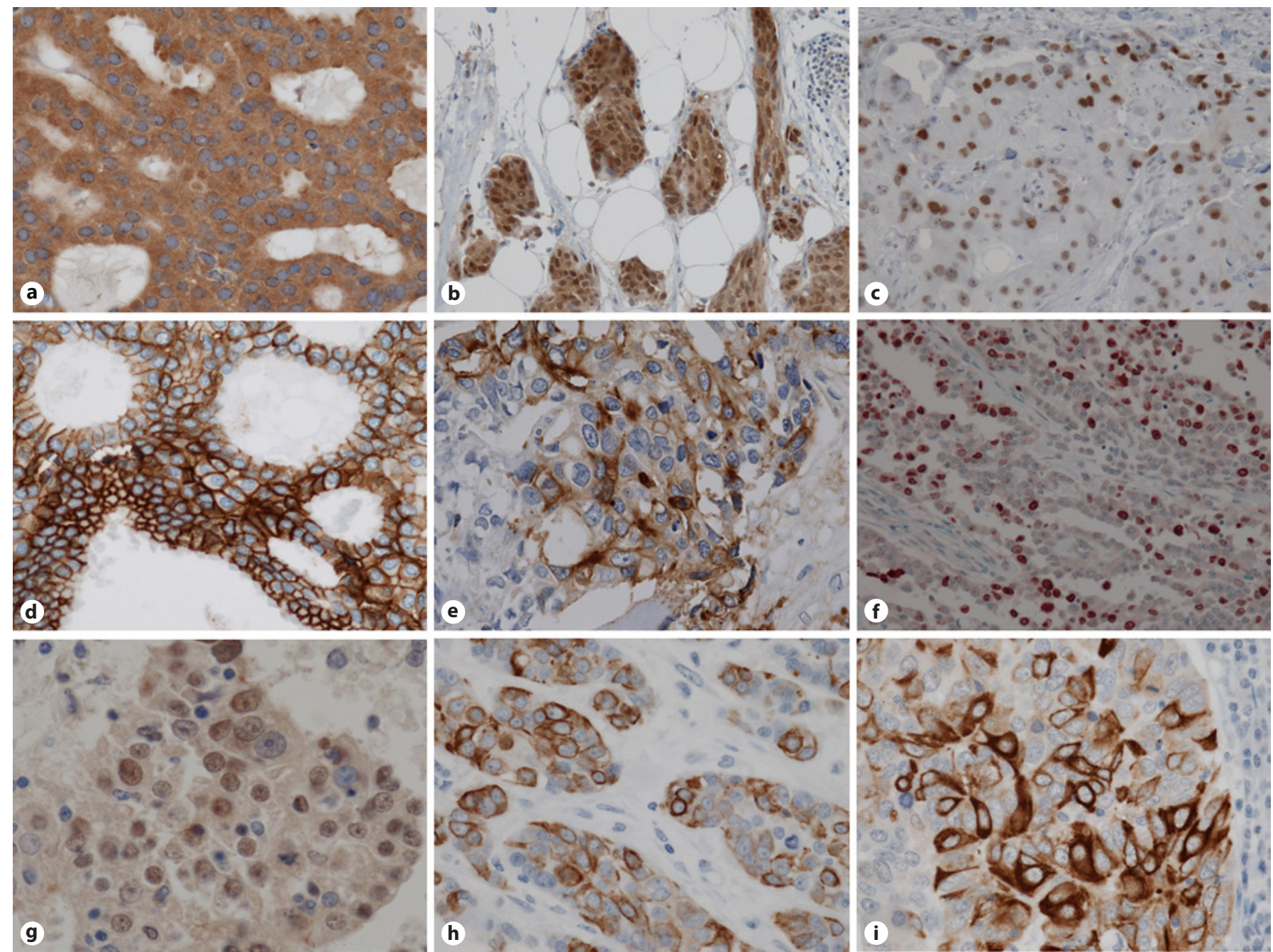

Fig. 1. a-e Immunohistochemical staining of mTOR signaling pathway molecules. a mTOR. b p-4EBP1. c HIF-1. d GLUT1. e GLUT3. f Immunohistochemical staining of the Ki67 antigen. $\mathbf{g - i}$ Localization of p-mTOR, as detected by immunohistochemistry, in the nucleus $(\mathbf{g})$, perinucleus $(\mathbf{h})$, and cytoplasm (i).

Fig. 2. Expression of each marker in triplenegative and non-triple-negative breast cancer. TNBC, triple-negative breast cancer. ${ }^{*} p<0.05$, statistically significant.

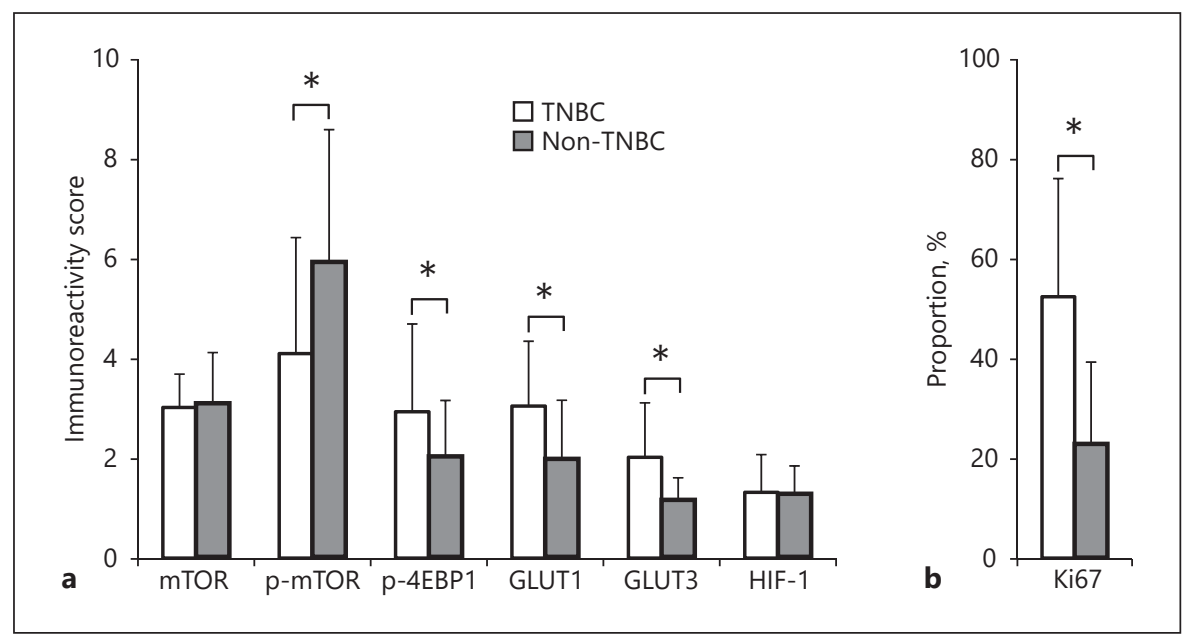


Table 3. Spearman's rank correlation among the expression of the molecules

\begin{tabular}{lcc}
\hline & $\rho$ & $p$ value \\
\hline mTOR vs. GLUT1 & 0.155 & 0.0961 \\
mTOR vs. GLUT3 & -0.0331 & 0.724 \\
mTOR vs. p-4EBP1 & -0.0081 & 0.931 \\
p-mTOR vs. GLUT1 & -0.0736 & 0.432 \\
p-mTOR vs. GLUT3 & -0.156 & 0.0951 \\
p-mTOR vs. p-4EBP1 & 0.111 & 0.237 \\
GLUT1 vs. GLUT3 & 0.461 & $<0.0001$ \\
GLUT1 vs. p-4EBP1 & 0.246 & 0.0079 \\
GLUT1 vs. HIF-1 & 0.336 & 0.0002 \\
GLUT3 vs. p-4EBP1 & 0.267 & 0.0037 \\
Ki67 vs. mTOR & -0.0001 & 0.998 \\
Ki67 vs. p-mTOR & -0.321 & 0.0004 \\
Ki67 vs. GLUT1 & 0.496 & $<0.0001$ \\
Ki67 vs. GLUT3 & 0.579 & $<0.0001$ \\
Ki67 vs. p-4EBP1 & 0.192 & 0.0395 \\
Ki67 vs. HIF-1 & 0.201 & 0.03 \\
\hline
\end{tabular}

Proportion of cells positive for Ki67. Immunoreactivity score for mTOR, p-mTOR, p-4EBP1, HIF-1, GLUT1, and GLUT3.

Table 4. Localization of $\mathrm{p}-\mathrm{mTOR}$ in cases of TNBC and nonTNCB

\begin{tabular}{lrlll}
\hline & $n$ & Nuclear & Perinuclear & Cytoplasmic \\
\hline TNBC & 35 & $5(14 \%)$ & $6(17 \%)$ & $24(68 \%)$ \\
Non-TNBC & 81 & $1(1 \%)$ & $19(23 \%)$ & $61(75 \%)$ \\
Total & 116 & $6(5 \%)$ & $25(22 \%)$ & $85(73 \%)$ \\
$p$ value & & 0.009 & 0.623 & 0.49 \\
\hline
\end{tabular}

Values express $n$ (\%). TNBC, triple-negative breast cancer.

\section{Localization of $m$ TOR and $p-m T O R$}

In all TNBC and non-TNBC cases, both mTOR and p-mTOR were assigned an immunoreactivity score of at least 1 . mTOR expression was confined to the cytoplasm. However, among the 35 TNBC cases, nuclear p-mTOR was expressed in $5(14 \%)$, perinuclear p-mTOR in 6 $(17 \%)$, and cytoplasmic p-mTOR in $24(68 \%)$. On the other hand, in the 81 non-TNBC cases, nuclear p-mTOR, perinuclear p-mTOR, and cytoplasmic p-mTOR were found in $1(1 \%), 19(23 \%)$, and $61(75 \%)$ cases, respectively (Fig. 1) (Table 4). Expression of GLUT1 and GLUT3 was found to be significantly higher in cases with nuclear p-mTOR expression than in those with perinuclear or cytoplasmic expression (Fig. 3a, 4).

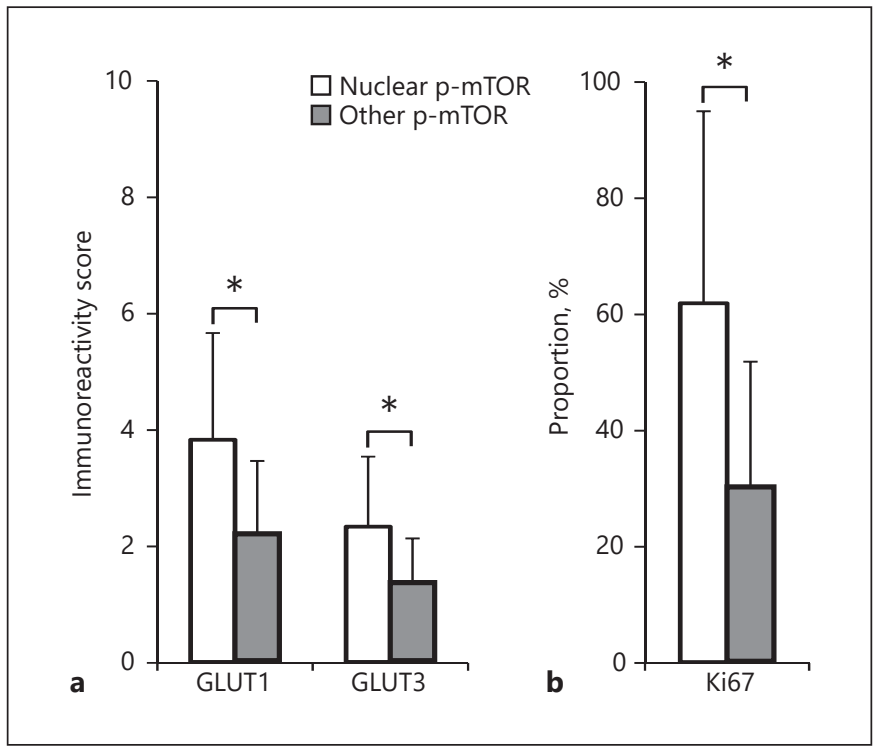

Fig. 3. Immunoreactivity scores and Ki67 proportion according to p-mTOR localization. ${ }^{*} p<0.05$, statistically significant.

\section{Relation with Proliferation Activity}

The proportion of Ki67-positive cells was significantly higher in TNBC cases than in non-TNBC cases (mean \pm SD: $52 \pm 24$ vs. $23 \pm 16 \%$; $p<0.0001$ ) (Fig. 2 b). The proportion of Ki67-positive cells correlated with the immunoreactivity scores for GLUT1 and GLUT3, but not with those for mTOR and p-mTOR (Table 3). In addition, the proportion of Ki67-positive cells was higher in the cases with nuclear $\mathrm{p}$-mTOR expression than in those with perinuclear or cytoplasmic expression (Fig. 3b).

\section{Discussion}

Therapeutic inhibition of the PI3K/mTOR pathway in breast cancer is an active area of research. Several ongoing studies are evaluating the effect of PI3K/mTOR inhibitors in TNBC, administered alone or in combination with chemotherapy [4]. Most previous studies that evaluated $\mathrm{p}$-mTOR expression in breast cancer did not consider the breast cancer subtype, with the exception of 3 studies that did describe p-mTOR expression in TNBC [14-16]. Ueng et al. [15] reported p-mTOR positivity in $72 \%$ of TNBC tumors from 177 patients. Bakarakos et al. [16] described a positive association between $\mathrm{p}$-mTOR expression and lymph node status and a negative impact of p-mTOR expression on overall survival. We evaluated the immunohistochemical expression of p-mTOR in 116 breast can- 

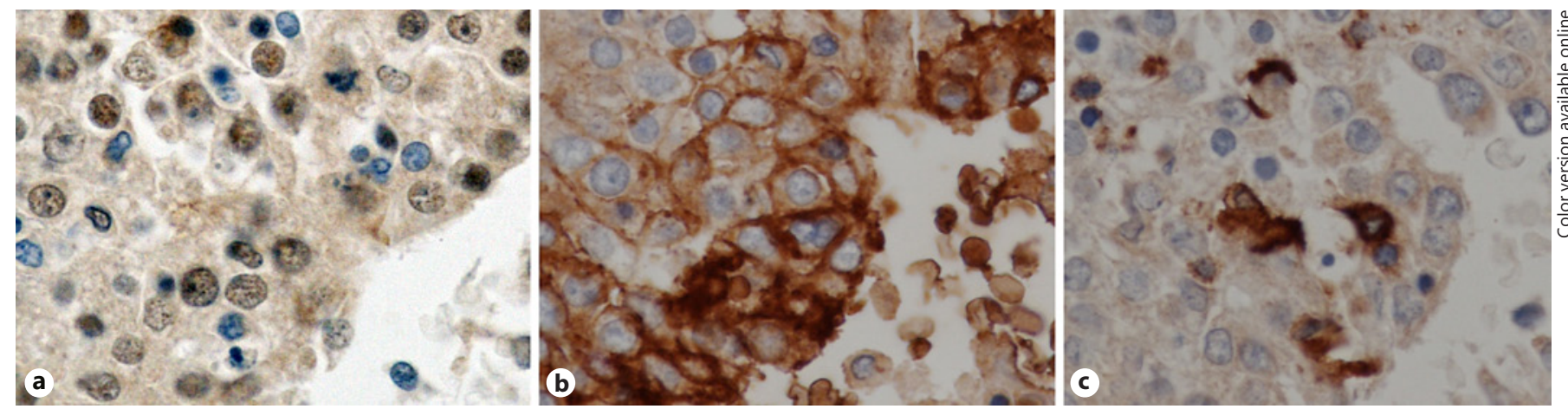

Fig. 4. Immunohistochemical staining of p-mTOR, GLUT1, and GLUT3 using sequential tissue sections from a case of TNBC. a pmTOR nuclear expression. b Diffuse strong expression of GLUT1. c Strong expression of GLUT3 in a portion of cancer cells.

cer specimens and found that 63 (54\%) had an immunoreactivity score $>6$. This suggests that $>50 \%$ of breast cancer patients are potential candidates for treatment with p-mTOR inhibitors. Our findings are corroborated by Walsh et al. [14], who detected increased p-mTOR expression in TNBC versus non-TNBC.

Our results regarding the relationship between $\mathrm{p}-\mathrm{mTOR}$ and GLUT expression appear contradictory when taking the mTOR signaling pathway into consideration. In the mTOR signaling pathway, increased expression of $\mathrm{p}$ mTOR causes increased expression of $\mathrm{p}-4 \mathrm{EBP} 1$ via HIF-1 $\alpha$, leading to increased GLUT expression $[2,12]$. On the other hand, in our data, although p-mTOR increased in nonTNBC versus TNBC, the $\mathrm{p}-4 \mathrm{EBP} 1$ expression was higher in TNBC than in non-TNBC. HIF-1a expression is controlled by mTOR in several ways and under different conditions, including hypoxia and mitogenic stimulation [17].

Other studies have suggested an HIF-1a-dependent regulation of GLUT1 [12] and GLUT3 [13]. We therefore examined the intracellular localization of p-mTOR. While mTOR localizes predominantly in the cytoplasm under steady-state conditions, cytoplasmic/nuclear shuttling of mTOR regulates 4EBP1 phosphorylation [18]. In this study, comparing the localization of p-mTOR between TNBC and non-TNBC cases, p-mTOR nuclear expression was more frequently observed in TNBC, which is consistent with a previous report by Walsh et al. [14]. In addition, the expression of p-mTOR in the nucleus correlated with that of GLUT1 and GLUT3. Our data indicate that p-mTOR in the nucleus may play an important role, particularly in TNBC.

Vazquez-Martin et al. [19] observed that nuclear enrichment of p-mTOR is closely related to the proliferative capacity of breast cancer cell lines. In our study, the ex- pression of Ki67 (reflecting proliferative activity) was positively correlated with that of GLUT1 $(\rho=0.496)$ and GLUT3 $(\rho=0.579)$. Ki67 index is an important factor when considering prognosis [20]. However, in this study, no direct relationship between prognosis and GLUT1 or GLUT3 expression could be demonstrated.

Although treatment of breast cancer with MTOR inhibitors has been reported [3,4], it may not be an effective therapeutic strategy at present. Furthermore, mTOR localization was not examined in previous clinical trials of mTOR inhibitors [3-5]. Our results may be helpful for identifying those patients most likely to benefit from mTOR inhibition by examining p-mTOR localization.

\section{Statement of Ethics}

The study protocol was approved by the Ethics Committee of Toho University School of Medicine (27119).

\section{Disclosure Statement}

There were no disclosures.

\section{References}

1 Podo F, Buydens LM, Degani H, Hilhorst R, Klipp E, Gribbestad IS, et al.; FEMME Consortium. Triple-negative breast cancer: present challenges and new perspectives. Mol Oncol. 2010 Jun;4(3):209-29.

2 Laplante M, Sabatini DM. mTOR signaling in growth control and disease. Cell. 2012 Apr; 149(2):274-93.

3 Lousberg L, Jerusalem G. Safety, Efficacy, and Patient Acceptability of Everolimus in the Treatment of Breast Cancer. Breast Cancer (Auckl). 2017 Jan;10:239-52. 
4 Basho RK, Gilcrease M, Murthy RK, Helgason T, Karp DD, Meric-Bernstam F, et al. Targeting the PI3K/AKT/mTOR pathway for the treatment of mesenchymal triple-negative breast cancer: evidence from a phase 1 trial of mTOR inhibition in combination with liposomal doxorubicin and bevacizumab. JAMA Oncol. 2017 Apr;3(4):509-15.

5 Costa RL, Han HS, Gradishar WJ. Targeting the $\mathrm{PI} 3 \mathrm{~K} / \mathrm{AKT} / \mathrm{mTOR}$ pathway in triple-negative breast cancer: a review. Breast Cancer Res Treat. 2018 Jun;169(3):397-406.

6 Holz MK, Ballif BA, Gygi SP, Blenis J. mTOR and S6K1 mediate assembly of the translation preinitiation complex through dynamic protein interchange and ordered phosphorylation events. Cell. 2005 Nov;123(4):569-80.

7 Rowinsky EK. Targeting the molecular target of rapamycin (mTOR). Curr Opin Oncol. 2004 Nov; 16(6):564-75.

8 Noh WC, Paik NS. Translational regulation: a novel target for breast cancer therapy. J Breast Cancer. 2003;6(1):8-14.

9 Fingar DC, Blenis J. Target of rapamycin (TOR): an integrator of nutrient and growth factor signals and coordinator of cell growth and cell cycle progression. Oncogene. 2004 Apr;23(18):3151-71.

10 Bernardi R, Guernah I, Jin D, Grisendi S, Alimonti A, Teruya-Feldstein J, et al. PML inhibits HIF-1alpha translation and neoangiogenesis through repression of mTOR. Nature. 2006 Aug;442(7104):779-85.
11 Gordan JD, Thompson CB, Simon MC. HIF and c-Myc: sibling rivals for control of cancer cell metabolism and proliferation. Cancer Cell. 2007 Aug;12(2):108-13.

12 Shackelford DB, Vasquez DS, Corbeil J, Wu S, Leblanc M, Wu CL, et al. mTOR and HIFlalpha-mediated tumor metabolism in an LKB1 mouse model of Peutz-Jeghers syndrome. Proc Natl Acad Sci USA. 2009 Jul; 106(27):11137-42.

13 Yu J, Li J, Zhang S, Xu X, Zheng M, Jiang G, et al. IGF-1 induces hypoxia-inducible factor 1a-mediated GLUT3 expression through PI3K/Akt/mTOR dependent pathways in PC12 cells. Brain Res. 2012 Jan;1430:18-24.

14 Walsh S, Flanagan L, Quinn C, Evoy D, McDermott EW, Pierce A, et al. mTOR in breast cancer: differential expression in triple-negative and non-triple-negative tumors. Breast. 2012 Apr;21(2):178-82.

15 Ueng SH, Chen SC, Chang YS, Hsueh S, Lin YC, Chien HP, et al. Phosphorylated mTOR expression correlates with poor outcome in early-stage triple negative breast carcinomas. Int J Clin Exp Pathol. 2012;5(8):806-13.

16 Bakarakos P, Theohari I, Nomikos A, Mylona E, Papadimitriou C, Dimopoulos AM, et al. Immunohistochemical study of PTEN and phosphorylated mTOR proteins in familial and sporadic invasive breast carcinomas. Histopathology. 2010 Jun;56(7):876-82.
17 Dunlop EA, Tee AR. Mammalian target of rapamycin complex 1: signalling inputs, substrates and feedback mechanisms. Cell Signal. 2009 Jun;21(6):827-35.

18 Kim JE, Chen J. Cytoplasmic-nuclear shuttling of FKBP12-rapamycin-associated protein is involved in rapamycin-sensitive signaling and translation initiation. Proc Natl Acad Sci USA. 2000 Dec;97(26):14340-5.

19 Vazquez-Martin A, Oliveras-Ferraros C, Bernadó L, López-Bonet E, Menendez JA. The serine 2481-autophosphorylated form of mammalian Target of Rapamycin (mTOR) is localized to midzone and midbody in dividing cancer cells. Biochem Biophys Res Commun. 2009 Mar;380(3):638-43.

20 Urruticoechea A, Smith IE, Dowsett M. Proliferation marker Ki-67 in early breast cancer. J Clin Oncol. 2005 Oct;23(28):7212-20.

21 Tsuda H, Akiyama F, Kurosumi M, Sakamoto G, Watanabe T; Japan National Surgical Adjuvant Study of Breast Cancer (NSAS-BC) Pathology Section. Establishment of histological criteria for high-risk node-negative breast carcinoma for a multi-institutional randomized clinical trial of adjuvant therapy. Jpn J Clin Oncol. 1998 Aug;28(8):486-91.

22 Elston CW, Ellis IO. Pathological prognostic factors in breast cancer. I. The value of histological grade in breast cancer: experience from a large study with long-term follow-up. Histopathology. 1991 Nov;19(5):403-10. 